\title{
IrRECIST No Baseline Disease
}

National Cancer Institute

\section{Source}

National Cancer Institute. irRECIST No Baseline Disease. NCI Thesaurus. Code C140318.

No target disease was identified at baseline and at follow-up the patient fails to meet criteria for irCR or irPD. 